\title{
Long-term disease control with triapine-based radiochemotherapy for patients with stage IB2-IIIB cervical
}

\section{cancer}

\author{
Charles A. Kunos ${ }^{1 *}$ and Tracy M. Sherertz ${ }^{2}$ \\ 1 Department of Radiation Oncology, Summa Cancer Institute, Summa Health System, Akron, OH, USA \\ 2 Department of Radiation Oncology, CASE Comprehensive Cancer Center, University Hospitals Case Medical Center, Case Western Reserve University School of \\ Medicine, Cleveland, OH, USA
}

\section{Edited by:}

Adam Paul Dicker, Thomas Jefferson University, USA

Reviewed by:

Loren K. Mell, University of California San Diego, USA

Aradhana Kaushal, National Cancer Institute, USA

\section{*Correspondence}

Charles A. Kunos, Department of Radiation Oncology, Summa Cancer Institute, G-90 161 North Forge Street, Akron, $\mathrm{OH}$ 44304, USA e-mail:kunosc@summahealth.org
Background: National Cancer Institute phase I \#7336 and phase II \#8327 clinical trials explored the safety and efficacy of triapine (NSC \#663249) added to cisplatin radiochemotherapy in untreated patients with advanced-stage cervical cancer. Triapine inhibits ribonucleotide reductase, the rate-limiting enzyme responsible for DNA-building deoxyribonucleotides, and thereby, enhances radiochemosensitivity by prolonging DNA repair time. Here, we report 3-year efficacy endpoints of pelvic locoregional relapse rate, disease-free, and overall survivals.

Methods: Eligible patients with bulky IB-IIIB cervical cancer underwent three-times weekly triapine ( 25 or $50 \mathrm{mg} / \mathrm{m}^{2}$ ), once-weekly cisplatin $\left(40 \mathrm{mg} / \mathrm{m}^{2}\right)$, and conventional daily pelvic radiation followed by brachytherapy. A cumulative incidence method estimated pelvic locoregional relapse rates. Disease-free survival was measured from radiochemotherapy start date to the date of first relapse or cancer-related death. Overall survival was measured from radiochemotherapy start date to the date of any-cause death. The Kaplan-Meier method estimated survivals.

Findings: Between 2006 and 2011, 24 untreated patients with cervical cancer met criteria for reporting in this study. A median 3.4 years of follow-up time (range, 0.3-7.6 years) has been observed. All had squamous cancers and the majority had either node-positive stage IB-IIA (33\%) or stage IIIB (42\%) disease. The 3-year pelvic locoregional relapse rate, disease-free survival, and overall survival were 4\% [95\% confidence interval (Cl), 0-20\%], $80 \%$ (95\% Cl: 71-89\%), and 82\% (95\% Cl: 74-90\%), respectively.

Interpretation:Triapine radiochemotherapy was safe, active, and effective in patients with untreated advanced-stage cervical cancer, worthy of randomized clinical trial study.

Keywords: triapine, cervical cancer, ribonucleotide reductase, radiation, cisplatin

\section{INTRODUCTION}

Incomplete radiochemotherapeutic treatment responses and poorer cancer-related survivals occur if human papilloma virus (HPV) and abnormal p53 signaling overactivates ribonucleotide reductase in cancers of the uterine cervix (1-8). Human cervical cancer cells overproduce de novo deoxyribonucleotides (the building blocks of DNA), overhaul radiochemotherapyinduced DNA damage more easily than other cancer cells, and outlive cytotoxic radiochemotherapy exposures (5-8). Pharmacological blockade of ribonucleotide reductase lowers enzyme catalytic activity, lengthens repair time of radiochemotherapyinduced DNA damage, and elicits cancer cell death (5-8). Enhanced cell lethality after ribonucleotide reductase inhibition may take advantage of a time-critical unmet high de novo deoxyribonucleotide demand during low de novo deoxyribonucleotide supply (9). In previously conducted clinical trials in untreated patients with advanced-stage cervical cancer, inhibitors of ribonucleotide reductase (e.g., hydroxyurea, 5 -fluorouracil, and gemcitabine) have improved radiochemotherapy efficacy (10-12). Another ribonucleotide reductase inhibitor, triapine (3-aminopyridine-2-carboxaldehyde thiosemicarbazone, NSC \#663249), has further boosted radiochemotherapeutic activity and efficacy in untreated patients with advanced-stage cervical cancer $(1,2)$.

Overall, two important anticancer strategies have demonstrated positive clinical outcomes in cervical cancer management: (a) ribonucleotide reductase inhibition $(1,2)$, and (b) angiogenesis inhibition $(13,14)$. This study of triapine radiochemotherapy protocols \#7336 and \#8327 was undertaken to report long-term clinical outcomes and toxicity in selected previously untreated stage IB2-IIIB cervical cancer patients nearly matching the Radiation Therapy Oncology Group protocol \#0417 cohort (13). 


\section{METHODS \\ PATIENT ELIGIBILITY}

For the triapine dose-finding phase I protocol \#7336, eligible patients had any histologically confirmed primary or recurrent pelvic gynecologic malignancy not amenable to curative surgery. For the triapine efficacy phase II protocol \#8327, eligible patients had squamous carcinoma, adenocarcinoma, or adenosquamous carcinoma staged IB2-IVB of the uterine cervix or staged II-IV of the vagina. Prior reports list detailed eligibility criteria $(1,2)$. Herein, we exclusively report on those enrolled patients with International Federation of Gynecologists and Obstetricians (FIGO) stage II or IIIB disease, or node-positive FIGO stage IB2 whose tumor size exceeded $5 \mathrm{~cm}$ (Figure 1; Table 1). All patients provided written informed consent. University Hospitals of Cleveland and Case Western Reserve University (Cleveland, OH, USA) institutional review board approvals were granted for these clinical trials. The Case Comprehensive Cancer Center of University Hospitals of Cleveland and Case Western Reserve University provided oversight for the data and safety monitoring plans.

\section{RADIATION, CISPLATIN CHEMOTHERAPY, AND TRIAPINE TREATMENT}

The details of dose-escalation and protocol therapy have been outlined previously $(1,2)$. Briefly, patients underwent three-times weekly triapine $\left[25\right.$ or $50 \mathrm{mg} / \mathrm{m}^{2}$ ( $1 \mathrm{~h}$ after radiation on days 1,3 , and 5) $]$ and once-weekly cisplatin $\left[40 \mathrm{mg} / \mathrm{m}^{2}\right.$ (prior to radiation on day 2)] chemotherapy during daily four-field pelvic radiation therapy (days 1 through 5 ). This schedule was repeated for five

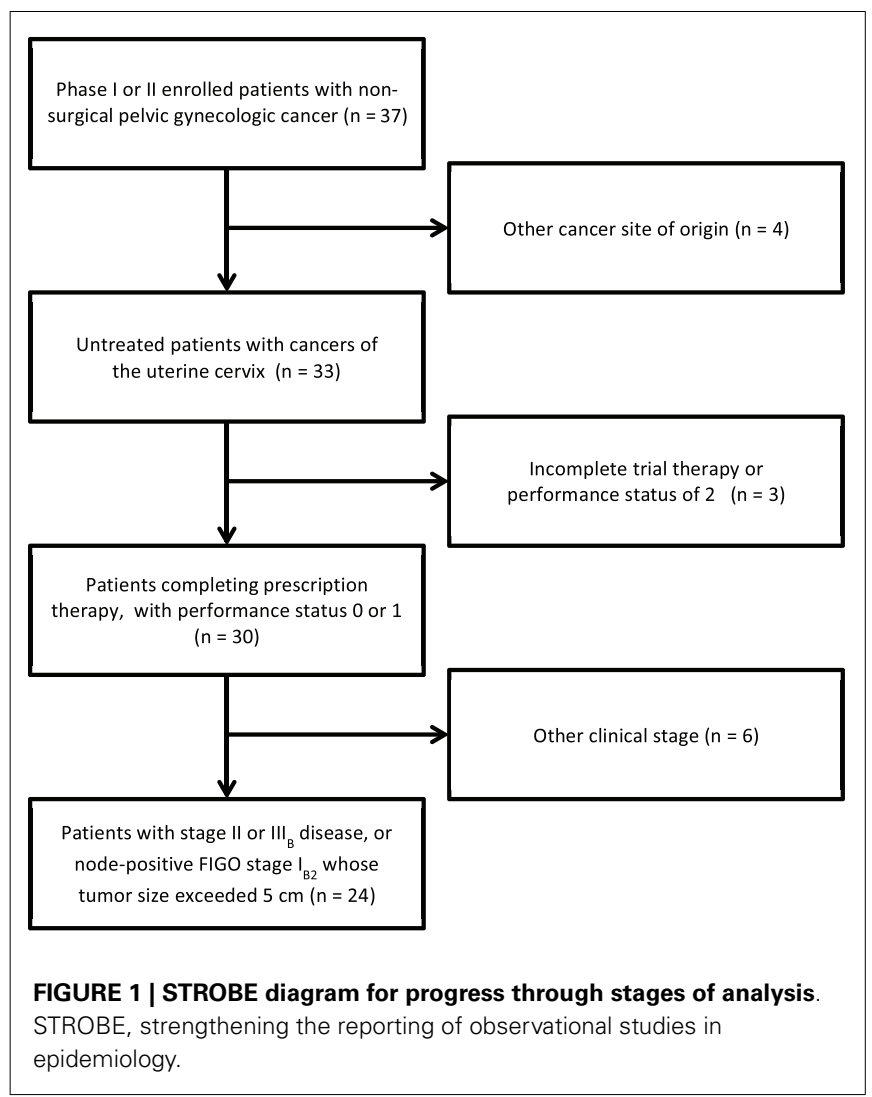

consecutive weeks (median $45 \mathrm{~Gy}$ ). An anteroposterior parametrial boost with central pelvic shielding (median 9 Gy) was given during week six, with any missed triapine doses allowed to be made up during this boost. Intracavitary low-dose-rate brachytherapy (median $40 \mathrm{~Gy}$ ) brought the median point A dose to $85 \mathrm{~Gy}$. No adjuvant therapy was administered in these trials.

\section{PATIENT ASSESSMENTS AND FOLLOW-UP}

Patients had physical examinations and hematologic, hepatic, and renal function blood testing and baseline computed tomography $(\mathrm{CT})$ or 2 - $\left[{ }^{18} \mathrm{~F}\right]$ fluoro-2-deoxy-D-glucose positron emission tomography scans (FDG PET/CT) within 28 days before the start of radiochemotherapy. Physical examinations, adverse event assessments (National Cancer Institute Common Terminology Criteria for Adverse Events, version 3.0), and blood work were repeated weekly. Posttherapy physical examinations and adverse event assessments were required at 1 month and at 3 months after completing all radiation therapy. Patients were followed generally every 3 months thereafter by one of the treating physicians. Three-month FDG PET/CT scans were mandatory on the phase II protocol \#8327 and optional on the phase I protocol \#7336.

Table 1 | Pretherapy patient and tumor characteristics $(n=24)$.

\begin{tabular}{|c|c|c|}
\hline \multicolumn{3}{|l|}{ Age (years) } \\
\hline Median & 57 & \\
\hline Range & $33-68$ & \\
\hline \multicolumn{3}{|l|}{ Race } \\
\hline Black or African American & 9 & $(38 \%)$ \\
\hline White & 15 & $(62 \%)$ \\
\hline \multicolumn{3}{|l|}{ Ethnicity } \\
\hline Hispanic or Latino & 2 & $(8 \%)$ \\
\hline Not Hispanic or Latino & 22 & $(92 \%)$ \\
\hline \multicolumn{3}{|l|}{ Performance status } \\
\hline 0 & 23 & $(96 \%)$ \\
\hline 1 & 1 & $(4 \%)$ \\
\hline \multicolumn{3}{|l|}{ Histology } \\
\hline Squamous & 24 & $(100 \%)$ \\
\hline Adenocarcinoma & 0 & $(0 \%)$ \\
\hline Adenosquamous & 0 & $(0 \%)$ \\
\hline \multicolumn{3}{|l|}{ Histologic grade } \\
\hline G1: well differentiated & 0 & $(0 \%)$ \\
\hline G2: moderately differentiated & 10 & $(42 \%)$ \\
\hline G3: poorly differentiated & 14 & $(58 \%)$ \\
\hline \multicolumn{3}{|l|}{ Lymph node status } \\
\hline Positive & 15 & $(63 \%)$ \\
\hline Negative & 9 & $(37 \%)$ \\
\hline \multicolumn{3}{|l|}{ FIGO stage } \\
\hline IB2 & 6 & $(25 \%)$ \\
\hline ॥A & 6 & $(25 \%)$ \\
\hline IIB & 2 & $(8 \%)$ \\
\hline IIIA & 0 & $(0 \%)$ \\
\hline IIIB & 10 & $(42 \%)$ \\
\hline
\end{tabular}

FIGO, international federation of gynecology and obstetrics. 


\section{STATISTICAL METHODS}

For this study, a pelvic locoregional relapse was defined as any disease occurring within the pelvic radiation treatment field. Extrapelvic distant disease relapse was defined as any new disease occurring outside of the radiation treatment field, inclusive of para-aortic lymph node relapses. Pathologic confirmation of relapse was not required. The rates of pelvic locoregional relapse were calculated as a proportion and with the $95 \%$ confidence interval (CI) determined with no continuity correction. Disease-free survival events were defined as cancer-related death, locoregional relapse, and extrapelvic relapse. Disease-free survival was measured from the start date of radiochemotherapy through the date of first relapse or cancer-related death. Overall survival events were deaths from any cause. Overall survival was measured from radiochemotherapy start date through the date of death. Diseasefree and overall survival were calculated by the method of Kaplan and Meier (15).

Toxicity endpoints were protocol-defined and scored according to National Cancer Institute Common Terminology Criteria for Adverse Events, version $3.0(1,2)$. Table 2 identifies protocoldefined adverse events happening during therapy or within the first 30 days posttherapy, and late adverse events occurring greater than 30 days posttherapy.

\section{RESULTS}

\section{PATIENTS}

Phase I accrual to protocol \#7336 occurred between May, 2006 and August, 2008. Phase II accrual to protocol \#8327 transpired between August, 2009, and November, 2011. Thirty-seven patients were recruited to these two protocols from a single institution; 33 patients had squamous, adenocarcinoma, or adenosquamous cancers of the uterine cervix of which 24 comprise this analysis (Figure 1). Of the 13 patients excluded from this analysis, 1 patient had stage IV uterine stromal sarcoma; 3 patients had vaginal cancer; 1 patient did not receive any protocol therapy; 1 patient had a

Table 2 | Protocol-defined triapine-attributed treatment adverse events occurring at any time $(n=24)$.

\begin{tabular}{|c|c|c|c|c|}
\hline \multirow[b]{3}{*}{ Category } & \multirow{2}{*}{\multicolumn{2}{|c|}{$\begin{array}{l}\text { Acute }^{\mathbf{a}} \\
\text { Grade }\end{array}$}} & \multirow{2}{*}{\multicolumn{2}{|c|}{$\begin{array}{l}\text { Late }^{\mathbf{b}} \\
\text { Grade }\end{array}$}} \\
\hline & & & & \\
\hline & 3 & 4 & 3 & 4 \\
\hline Methemoglobinemia & 0 & 0 & 0 & 0 \\
\hline Blood/bone marrow & 2 & 0 & 0 & 0 \\
\hline Cardiovascular (general) & 0 & 0 & 0 & 0 \\
\hline Gastrointestinal & 0 & 0 & 1 & 0 \\
\hline Neurology & 1 & 0 & 0 & 0 \\
\hline Renal (electrolyte) & 1 & 2 & 0 & 0 \\
\hline Genitourinary & 0 & 0 & 2 & 0 \\
\hline Worst non-hematologic & $2(8 \%)$ & $2(8 \%)$ & $3(13 \%)$ & $0(0 \%)$ \\
\hline Worst overall & $4(17 \%)$ & $2(8 \%)$ & $3(13 \%)$ & $0(0 \%)$ \\
\hline
\end{tabular}

${ }^{a}$ Occurring on-treatment or within 30 days of any protocol treatment.

${ }^{b}$ Occurring $>30$ days after any protocol treatment. pretherapy Zubrod performance status of 2; 1 patient had clinical stage IIIB but radiographic stage IVB disease and died from an iatrogenic Mallory-Weiss tear prior to brachytherapy; 2 patients had stage IB2 node negative cervical cancer; 1 patient had stage IVA cervical cancer; and 3 patients had clinical stage IVB cervical cancer. Pretherapy demographic and tumor characteristics for the remaining 24 patients are listed in Table 1. Median followup time was 3.4 years (range, 0.3-7.6 years). Median follow-up among survivors was 3.8 years (range, 2.2-7.6 years). For the 24 patients reported herein, the median age was 57 years (range, 3368 years). The majority of the 24 patients had a pretherapy Zubrod performance status of $0(96 \%)$ and either node-positive stage IBIIA $(n=8,33 \%)$ or stage IIIB $(n=10,42 \%)$ disease. Although these protocols included non-squamous cervical cancers, all 24 patients under study here had squamous cell cancers of the uterine cervix. Fifteen (63\%) had HPV subtype-16 and two (8\%) had HPV subtype-18. Seven tumors lacked $(n=5)$ or had unknown $(n=2)$ HPV status by polymerase chain reaction-restriction fragment length polymorphism.

\section{ADVERSE EVENTS}

Adverse events attributable to triapine administration or biologic effect were low in these 24 patients (Table 2). Symptomatic methemoglobinemia [i.e., methemoglobin concentration $>20 \%$ associated with dyspnea $(1,2)]$ did not occur in patients in either protocol. Attributable grade 3 or 4 toxicity occurred ontreatment or within 30 days of any protocol therapy in six patients $(25 \%)$. Attributable late $>30$-day posttherapy toxicity included two (8\%) incidences of grade 3 vaginitis and one (4\%) incidence of enterovaginal fistula. No triapine radiochemotherapy treatment administration-related deaths took place.

\section{TREATMENT COMPLIANCE}

Compliance with clinical trial protocol-specified radiation and chemotherapy administration was considered satisfactory. Three National Cancer Institute-monitored audits confirmed data timeliness and quality. A total of 342 (95\%) of an expected 360 triapine infusions occurred in the 24 patients studied here; there were no triapine infusion-related adverse events.

\section{EFFICACY}

The 3-year pelvic locoregional relapse rate, disease-free survival, and overall survival were $4 \%$ (95\% CI: $0-20 \%), 80 \%$ (95\% CI: $71-89 \%$ ), and $82 \%$ (95\% CI: 74-90\%), respectively. Figure 2 plots disease-free survival and overall survival curves. Nineteen (79\%) patients were without treatment failure at the time of last followup. Among women in this particular cohort, a single right pelvic sidewall relapse was detected at 8 months after the start of triapine radiochemotherapy. In this patient, a 3-month FDG PET/CT suggested an incomplete pelvic lymph node metabolic response (2). At surgery conducted to palliate non-treatment-related pain, a site of viable extranodal disease was removed surgically from the right pelvic sidewall. Four extrapelvic relapses (i.e., outside the radiation portal) have been recorded: two (8\%) para-aortic lymph node relapses; one (4\%) abdominal wall relapse; and one (4\%) left supraclavicular lymph node relapse. Five $(20 \%)$ deaths have occurred. Two ( $8 \%$ ) of the deaths were attributed to cancer-related factors. 

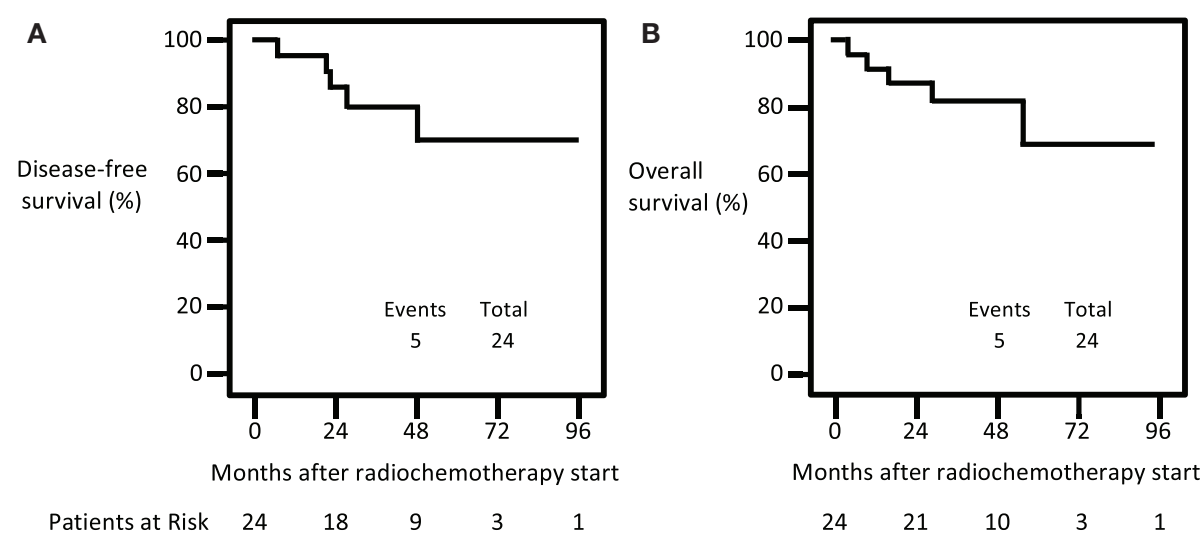

FIGURE 2 | Kaplan-Meier survival curves for disease-free survival (A) and overall survival (B).

\section{DISCUSSION}

Triapine added to cisplatin radiochemotherapy resulted in a low treatment-related adverse event rate and produced a 3-year disease-free survival rate of $80 \%$ (95\% CI: 71-89\%). These two important clinical trials demonstrate how the supply and demand economics of deoxyribonucleotides affect the degree to which cervical cancers respond to conventional treatment. The majority of cervical cancers have (1) HPVs that hijack ribonucleotide reductase to mint deoxyribonucleotides for replication of viral DNA, or (2) mutations in p53 that abrogate cell cycle restriction checkpoints, free-up ribonucleotide reductase to increase deoxyribonucleotide payout, and replicate DNA unchecked (9). As a consequence of either biologic phenomenon, ribonucleotide reductase subunits $\mathrm{M} 2$ and $\mathrm{M} 2 \mathrm{~b}$ are abundant in cervical cancer cells $(3,4)$. In this biologic state, ribonucleotide reductase M2 and M2b subunits saturate its catalytic M1 subunit $(3,4)$, which leads to overproduction of deoxyribonucleotides $(5,6)$. Because cervical cancers are rich in active ribonucleotide reductase, which can facilitate DNA damage repair $(3,4)$, patients with (over)active ribonucleotide reductase might have a less than ideal radiochemotherapy treatment response. And yet when ribonucleotide reductase activity is blocked, radiochemotherapy-induced DNA damage persists, cancer cell death occurs, and cervical cancer disease becomes better controlled. Perhaps these concepts are best demonstrated by the $4 \%$ rate of pelvic locoregional treatment failure among previously untreated patients enrolled on these two radiochemotherapy trials using triapine to inhibit cervical cancer cell ribonucleotide reductase. Because of these intriguing findings, a National Cancer Institute Cancer Therapy Evaluation Program randomized phase II trial currently tests triapine radiochemotherapy versus cisplatin radiochemotherapy in untreated patients with locally advanced-stage cervical cancer (NCT01835171).

The antitumor consequence of ribonucleotide reductase inhibition has not been the only advancement in cervical cancer treatment. Angiogenesis inhibition has emerged as an effective alternative anticancer treatment strategy for patients with cancers of the uterine cervix and may serve to frame the triapine radiochemotherapy results reported herein. Inactivation of angiogenesis effectors reduces small blood vessel growth, blocks new blood vessel formation, and restores tumor blood nutrient and oxygen supply so that treatments such as chemotherapy and radiation are more deadly to cancer cells $(16,17)$. Gynecologic Oncology Group protocol \#227C studied single-agent bevacizumab (15 mg/kg every 21 days) as an angiogenesis inhibitor in women with recurrent or metastatic cervical cancer and found a $24 \%$ rate of disease-free survival at 6 months (18). Phase III testing involved adding bevacizumab ( $15 \mathrm{mg} / \mathrm{kg}$ every 21 days) to cisplatin-paclitaxel or cisplatin-topotecan chemotherapy in patients with metastatic, persistent, or recurrent cervical cancer on Gynecologic Oncology Group protocol \#240 (14). Bevacizumab was administered until disease progression or manifestation of unacceptable toxicity. On this trial, a noteworthy 3.7-month improvement in median overall survival (17 vs. 13.3 months) was observed. Also, the Radiation Therapy Oncology Group embarked upon protocol \#0417 to evaluate the addition of three bevacizumab doses $(10 \mathrm{mg} / \mathrm{kg})$ to weekly cisplatin radiochemotherapy in 49 patients with bulky stage IB2-IIIB cervical cancer (13). No maintenance bevacizumab was administered. The trial showed a 3-year overall survival of $81 \%$ (95\% CI: 67-90\%), disease-free survival of $69 \%$ (95\% CI: 54-80\%), and cumulative incidence of locoregional relapse of $23 \%$ (95\% CI: $11-35 \%)$. The bevacizumab and cisplatin radiochemotherapy grade 3 and grade 4 worst overall toxicity rates were 27 and 10\%, respectively.

Because of the exciting clinical outcomes for these two ribonucleotide reductase inhibition clinical trials, a randomized phase II trial of cisplatin radiochemotherapy alone or with coadministered triapine has been put forward for consideration in the National Cancer Institute National Clinical Trials Network program.

\section{RESEARCH IN CONTEXT Systematic review}

Our manuscript reports new 3-year safety and efficacy data from clinical trials conducted with triapine radiochemotherapy in untreated patients with advanced-stage IB2-IIIB cancers of the uterine cervix. We searched PubMed with the terms "triapine," "radiation," "cervical cancer," and "clinical trial" for publications between January 1, 1999, and May 1, 2014. Only the original single 
institution phase I (1) and phase II (2) trials were found - with neither study providing more than 18-month clinical outcome data. We broadened our publication search to include "bevacizumab," given a publication discussing improved survival with bevacizumab in patients with advanced cervical cancer (14). These three bevacizumab clinical trials were selected to frame the context of our new long-term triapine radiochemotherapy clinical trial data $(13,14,18)$.

\section{Interpretation}

Three-year clinical trial outcomes after three-times weekly triapine added to cisplatin radiochemotherapy in patients with untreated locally advanced-stage cervical cancer demonstrate efficacy results that warrant further clinical trial evaluation. A randomized phase II clinical trial currently recruits untreated patients with stage IBIVA cancers of the uterine cervix to cisplatin radiochemotherapy with or without triapine (NCT01835171).

\section{AUTHOR CONTRIBUTIONS}

Charles A. Kunos and Tracy M. Sherertz contributed to the data collection and drafting of this manuscript. This manuscript has been seen, read, and agreed upon in its content by both designated authors.

\section{ACKNOWLEDGMENTS}

This work was supported originally by National Institutes of Health grants (U01 CA62502 and P30 CA43703-17). Clinical trial registry: NCT00335998 and NCT00941070 (clinicaltrials.gov).

\section{REFERENCES}

1. Kunos C, Waggoner S, Von Gruenigen V, Eldermire E, Pink J, Dowlati A, et al. Phase I trial of intravenous 3-aminopyridine-2-carboxaldehyde thiosemicarbazone (3-AP, NSC \#663249) in combination with pelvic radiation therapy and weekly cisplatin chemotherapy for locally advanced cervical cancer. Clin Cancer Res (2010) 16(4):1298-306. doi:10.1016/j.ygyno.2013.04.019

2. Kunos C, Radivoyevitch T, Waggoner S, DeBernardo R, Zanotti K, Resnick K, et al. Radiochemotherapy plus 3-aminopyridine-2-carboxaldehyde thiosemicarbazone (3-AP, NSC \#663249) in advanced-stage cervical and vaginal cancers. Gynecol Oncol (2013) 130(1):75-80. doi:10.1016/j.ygyno.2013.04.019

3. Kunos C, Winter K, Dicker A, Small WJ, Abdul-Karim FW, Dawson D, et al. Ribonucleotide reductase expression in cervical cancer: a radiation therapy oncology group translational science analysis. Int J Gynecol Cancer (2013) 23(4):615-21. doi:10.1097/IGC.0b013e31828b4eb5

4. Kunos C, Radivoyevitch T, Kresak A, Dawson D, Jacobberger J, Yang B, et al. Elevated ribonucleotide reductase levels associate with suppressed radiochemotherapy response in human cervical cancers. Int J Gynecol Cancer (2012) 22(9):1463-9. doi:10.097/IGC.0b013e318270577f

5. Kunos C, Chiu S, Pink J, Kinsella T. Modulating radiation resistance by inhibiting ribonucleotide reductase in cancers with virally or mutationally silenced p53 protein. Radiat Res (2009) 172(6):666-76. doi:10.1667/RR1858.1

6. Kunos C, Radivoyevitch T, Pink J, Chiu S, Stefan T, Jacobberger J, et al. Ribonucleotide reductase inhibition enhances chemoradiosensitivity of human cervical cancers. Radiat Res (2010) 174(5):574-81. doi:10.1667/RR2273.1

7. Kunos C, Colussi V, Pink J, Radivoyevitch T, Oleinick N. Radiosensitization of human cervical cancer cells by inhibiting ribonucleotide reductase: enhanced radiation response at low dose rates. Int J Radiat Oncol Biol Phys (2011) 80(4):1198-204. doi:10.1016/j.ijrobp.2011.01.034

8. Kunos C, Ferris G, Pyatka N, Pink J, Radivoyevitch T. Deoxynucleoside salvage facilitates DNA repair during ribonucleotide reductase blockade in human cervical cancers. Radiat Res (2011) 176(4):425-33. doi:10.1667/RR2556.1

9. Kunos C, Radivoyevitch T. Molecular strategies of deoxynucleotide triphosphate supply inhibition used in the treatment of gynecologic malignancies. Gynecol Obstetric (2011) S4:001. doi:10.4172/2161-0932.S4-001

10. Rose PG, Bundy BN, Watkins EB, Thigpen JT, Deppe G, Maiman MA, et al. Concurrent cisplatin-based radiotherapy and chemotherapy for locally advanced cervical cancer. N Engl J Med (1999) 340(15):1144-53. doi:10.1056/ NEJM199904153401502

11. Whitney CW, Sause W, Bundy BN, Malfetano JH, Hannigan EV, Fowler WC Jr et al. Randomized comparison of fluorouracil plus cisplatin versus hydroxyurea as an adjunct to radiation therapy in stage IIB-IVA carcinoma of the cervix with negative para-aortic lymph nodes: a Gynecologic Oncology Group and Southwest Oncology Group study. J Clin Oncol (1999) 17(5):1339-48.

12. Duenas-Gonzalez A, Zarba J, Patel F, Alcedo J, Beslija F, Casanova L, et al. A phase III, open-label, randomized study comparing concurrent gemcitabine plus cisplatin and radiation followed by adjuvant gemcitabine and cisplatin versus concurrent cisplatin and radiation in patients with stage IIB to IVA carcinoma of the cervix. J Clin Oncol (2011) 29(13):1678-85. doi:10.1200/JCO.2009.25. 9663

13. Schefter T, Winter K, Kwon J, Stuhr K, Balaraj K, Yaremko B, et al. RTOG 0417: efficacy of bevacizumab in combination with definitive radiation therapy and cisplatin chemotherapy in untreated patients with locally advanced cervical carcinoma. Int J Radiat Oncol Biol Phys (2014) 88(1):101-5. doi:10.1016/j.ijrobp. 2013.10.022

14. Tewari KS, Sill MW, Long HJ III, Penson RT, Huang H, Ramondetta LM, et al. Improved survival with bevacizumab in advanced cervical cancer. $N$ Engl J Med (2014) 370(8):734-43. doi:10.1056/NEJMoa1309748

15. Kaplan E, Meier P. Nonparametric estimation from incomplete observations. J Am Stat Assoc (1958) 53:457-81. doi:10.1080/01621459.1958.10501452

16. Shord S, Bressler L, Tierney L, Cuellar S, George A. Understanding and managing the possible adverse effects associated with bevacizumab. Am J Health Syst Pharm (2009) 66(11):999-1013. doi:10.2146/ajhp080455

17. Goel S, Duda D, Xu L, Munn L, Boucher Y, Fukumura D, et al. Normalization of the vasculature for treatment of cancer and other diseases. Physiol Rev (2011) 91:1071-121. doi:10.1152/physrev.00038.2010

18. Monk B, Sill M, Burger R, Gray H, Buekers T, Rotman L. Phase II trial of bevacizumab in the treatment of persistent or recurrent squamous cell carcinoma of the cervix: a gynecologic oncology group study. J Clin Oncol (2009) 27(7):1069-74. doi:10.1200/JCO.2008.18.9043

Conflict of Interest Statement: The authors declare that the research was conducted in the absence of any commercial or financial relationships that could be construed as a potential conflict of interest.

Received: 08 May 2014; accepted: 30 June 2014; published online: 24 July 2014. Citation: Kunos CA and Sherertz TM (2014) Long-term disease control with triapinebased radiochemotherapy for patients with stage IB2-IIIB cervical cancer. Front. Oncol. 4:184. doi: 10.3389/fonc.2014.00184

This article was submitted to Radiation Oncology, a section of the journal Frontiers in Oncology.

Copyright $\odot 2014$ Kunos and Sherertz. This is an open-access article distributed under the terms of the Creative Commons Attribution License (CC BY). The use, distribution or reproduction in other forums is permitted, provided the original author (s) or licensor are credited and that the original publication in this journal is cited, in accordance with accepted academic practice. No use, distribution or reproduction is permitted which does not comply with these terms. 